\title{
ARTICLE OPEN \\ Accelerated tooth movement in Rsk2-deficient mice with impaired cementum formation
}

\author{
Cita Nottmeier ${ }^{1}$, Maximilian G. Decker ${ }^{1}$, Julia Luther ${ }^{2}$, Simon von Kroge ${ }^{2}$, Bärbel Kahl-Nieke ${ }^{1}$, Michael Amling ${ }^{2}$, Thorsten Schinke ${ }^{2}$, \\ Julian Petersen ${ }^{2}$ and Till Koehne ${ }^{1}$
}

Coffin-Lowry-Syndrome (CLS) is a X-linked mental retardation characterized by skeletal dysplasia and premature tooth loss. We and others have previously demonstrated that the ribosomal S6 kinase RSK2, mutated in CLS, is essential for bone and cementum formation; however, it remains to be established whether RSK2 plays also a role in mechanically induced bone remodeling during orthodontic tooth movement (OTM). We, therefore, performed OTM in wild-type (WT) mice and Rsk2-deficient mice using Nitinol tension springs that were fixed between the upper left molars and the incisors. The untreated contralateral molars served as internal controls. After 12 days of OTM, the jaws were removed and examined by micro-computed tomography ( $\mu C T$ ), decalcified histology, and immunohistochemistry. Our analysis of the untreated teeth confirmed that the periodontal phenotype of Rsk2deficient mice is characterized by alveolar bone loss and hypoplasia of root cementum. Quantification of OTM using $\mu C T$ revealed that OTM was more than two-fold faster in Rsk2-deficient mice as compared to WT. We also observed that OTM caused alveolar bone loss and root resorptions in WT and Rsk2-deficient mice. However, quantification of these orthodontic side effects revealed no differences between WT and Rsk2-deficient mice. Taken together, Rsk2 loss-of-function accelerates OTM in mice without causing more side effects.

International Journal of Oral Science (2020)12:35

; https://doi.org/10.1038/s41368-020-00102-4

\section{INTRODUCTION}

The growth factor regulated ribosomal S6 kinase RSK2 (RPS6KA3) belongs to a group of widely expressed serine/threonine kinases, that are important for growth, survival, and proliferation of cells. ${ }^{1-3}$ RSK2 operates at the distal end of the Ras/Map kinase pathway and phosphorylates different substrates, such as cAMP response element-binding protein (CREB), c-Fos, activating transcription factor 4 (ATF4), and estrogen receptor alpha (ERa). ${ }^{4-6}$ CREB, ATF4, and $E R a$ are known to induce differentiation of bone-forming osteoblasts, ${ }^{4,7-12}$ whereas c-Fos is required for differentiation of bone-resorbing osteoclasts. ${ }^{13}$ RSK2, therefore, plays a pivotal role in bone turnover.

The role of RSK2 in bone homeostasis was deciphered by skeletal analyses of humans with Coffin-Lowry syndrome (CLS), carrying inactivating mutations in the $R s k 2$ gene, and by analyses of the corresponding Rsk2-deficient mouse model. Importantly, oral and dental findings in CLS patients also suggest a central role of RSK2 during dental development. The oral phenotype of CLS patients is characterized by hypodontia, microdontia, delayed eruption, and premature tooth loss. ${ }^{14-17}$ Employing Rsk2-deficient mice we have previously demonstrated that the premature tooth loss of CLS patients can be attributed to a cell-autonomous defect in cementoblast activity causing hypoplasia of root cementum, detachment of periodontal fibers, and subsequent alveolar bone loss. ${ }^{18}$ These findings were consistent with case reports describing that prematurely lost teeth of CLS patients had a reduced layer of root cementum. ${ }^{14,16}$
Despite its key function for bone and periodontal homeostasis, it is unknown whether RSK2 plays a role in orthodontic tooth movement (OTM) and the development of OTM side effects such as external root resorptions. The reasons for the occurrence of these root resorptions are far from being understood, but recent studies suggest that genetics plays a decisive role in its development. ${ }^{19-24}$ For instance, loss of bone sialoprotein in mice caused extensive root resorptions due to defective mineralization of the acellular cementum layer. ${ }^{25}$ Moreover, other proteins expressed in the cementum layer were found to have protective effects against root resorption. ${ }^{26}$ The cementum layer is therefore regarded as a barrier against root resorption. $^{27}$

Given the nonredundant role of RSK2 in bone and cementum formation we, therefore, asked how lack of RSK2 function in mice affects OTM and the emergence of root resorptions in vivo.

\section{RESULTS}

Loss of Rsk2 function accelerates tooth movement in mice We first analyzed the maxillary teeth of WT and $R s k 2^{-/ y}$ mice that were not subjected to OTM (OTM-) (Fig. 1a, upper panels). Our $\mu C T$ analysis revealed that the exposed root area, a surrogate measure for alveolar bone loss, was significantly larger in $R s k 2^{-/ y}$ mice as compared to that of WT mice (Fig. 1b). This observation in combination with the existence of an additional tooth in some of the $R s k 2^{-/ y}$ mice confirmed our previous analysis of mandibular

\footnotetext{
${ }^{1}$ Department of Orthodontics, University Medical Center Hamburg-Eppendorf, Hamburg, Germany and ${ }^{2}$ Institute of Osteology and Biomechanics, University Medical Center Hamburg-Eppendorf, Hamburg, Germany

Correspondence: Till Koehne (tkoehne@uke.de)
}

Received: 14 July 2020 Revised: 8 October 2020 Accepted: 4 November 2020

Published online: 23 December 2020 

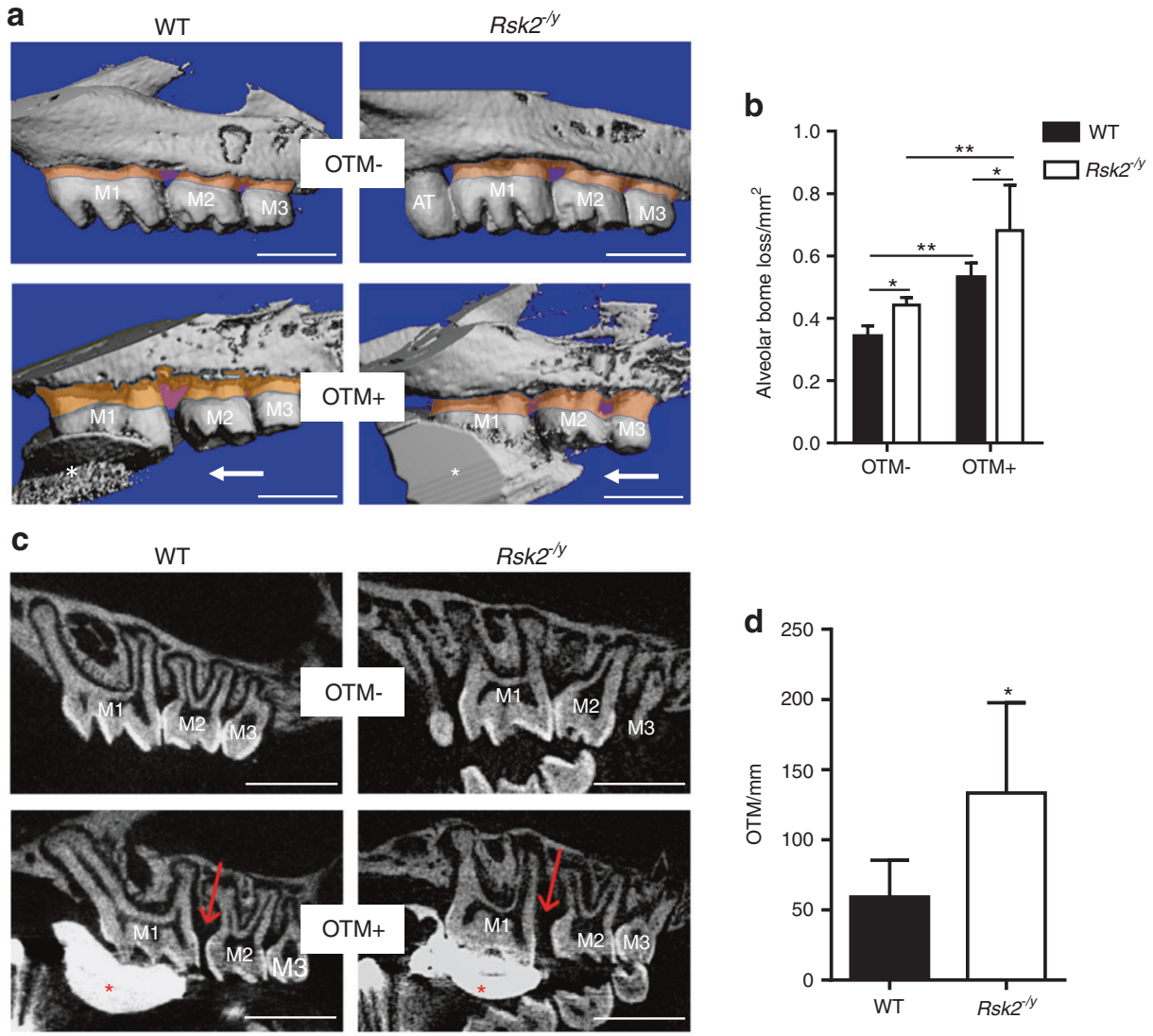

Fig. 1 Loss of Rsk2 function leads to faster tooth movement in mice. a Three-dimensional $\mu$-CT reconstruction of maxillary teeth from 12 weeks old $R s k 2^{-/ y}$ mice and wild-type littermates. Upper panels show untreated teeth (OTM-) and lower panels show teeth after orthodontic tooth movement (OTM+). The orange area indicates the horizontal alveolar bone loss, measured from enamel-cementumjunction to the alveolar bone crest. White arrows indicate the direction of the applied force. The scanning artifacts caused by the light-curing resin (white asterisks) are outside the region of interests and do not affect the measurements. M1, first molar; M2, second molar; M3, third molar; AT, additional tooth; scale bars $=1 \mathrm{~mm}$. b Quantification of the area of alveolar bone loss on the untreated (OTM-) and treated (OTM + ) side in $R s k 2^{-1 y}(n=4)$ and WT mice $(n=5)$. Values are means \pm SD. $\left({ }^{*} P<0.05,{ }^{* *} P<0.01\right)$ c $\mu$-CT cross-sections through M1-M3 showing the approximal gap after orthodontic tooth movement. Scale bars $=1 \mathrm{~mm}$. d Quantification of the intercoronal distance between $\mathrm{M} 1$ and $\mathrm{M} 2$ after 12 days of OTM in $R s k 2^{-/ y}$ and WT mice. ${ }^{*} P<0.05$

teeth from $R s k 2^{-/ y}$ mice. ${ }^{18}$ We next analyzed the alveolar bone of $R s k 2^{-/ y}$ and WT mice after OTM. We observed that OTM caused a loss of alveolar bone in Rsk2-/y and WT mice (Fig. 1a, lower panels). This OTM-induced increase of alveolar bone loss was statistically significant in both groups. To determine the amount of OTM we next analyzed the crowns of the first and second molars on $\mu C T$ cross-sections (Fig. 1c). The separation of the crowns after OTM was more apparent in $R s k 2^{-1 y}$ mice as compared to those of WT (Fig. 1c, lower panels). In fact, quantification of the gap between the two crowns revealed that OTM was more than twofold faster in $R s k 2^{-/ y}$ mice (Fig. 1d).

Periodontal response to OTM in $R s k 2^{-/ y}$ mice

We next performed decalcified histology to analyze the periodontal response to OTM in $R s k 2^{-1 y}$ and WT mice (Fig. 2a, b). Our analysis of teeth without tooth movement (OTM-) revealed no clear differences between $R s k 2^{-1 y}$ and WT mice. Only the acellular cementum layer in $R s k 2^{-/ y}$ mice appeared thinner as compared to those of WT mice (Fig. 2a, lower panels). In line with the $\mu C T$ evaluation we observed that the OTM-induced separation between the first molar (M1) and the second molar (M2) was wider in $R s k 2^{-1 y}$ mice as compared to that of WT (Fig. 2b). The OTM-induced gap between the teeth in $R s k 2^{-/ y}$ and WT mice caused food impaction and inflammatory hyperplasia of the sulcular epithelium associated with an accumulation of lymphocytes in the subepithelium (Fig. 2b, middle and lower panels).
This edematous swelling is significantly increased in both groups after the treatment (Fig. 2c).

To further investigate the soft tissue response to OTM, we next analyzed zones of OTM-induced tissue pressure and tissue tension in Rsk2-/y and WT mice (Fig. 3). We here focused on the distal root of the $M 1$ where the periodontal ligament (PDL) is exposed to tension on the distal root surface (Fig. 3a-c) and on the mesial root surface to pressure (Fig. 3d-f). Our analysis of untreated teeth revealed that most of the alveolar bone in $R s k 2^{-/ y}$ and WT mice was covered by bone lining cells and only a few active osteoblasts were evident (Fig. 3b). In contrast, after OTM we observed on the tension side multiple active, secreting osteoblasts on the alveolar bone surface in both $R s k 2^{-/ y}$ and WT mice. The observation of cement lines further indicated OTMinduced bone formation. Conversely, our histological analysis of the pressure side revealed signs of bone resorption after OTM as indicated by the presence of osteoclasts in Rsk2 $2^{-1 y}$ and WT mice (Fig. 3f). To better visualize these osteoclasts, we performed a staining with tartrate-resistant acid phosphatase (TRAP). Whereas no osteoclasts were evident in the PDL of teeth that were not subjected to tooth movement (Fig. 3h), multiple TRAP-positive cells were found in the pressure zones of teeth from $R s k 2^{-/ y}$ and WT mice after OTM. These TRAP-positive cells were evident not only on the alveolar bone surface but also within the PDL and on the root surface near root resorptions (Fig. 3i). Quantification of TRAP-positive cells showed a significant increase in both groups 
a
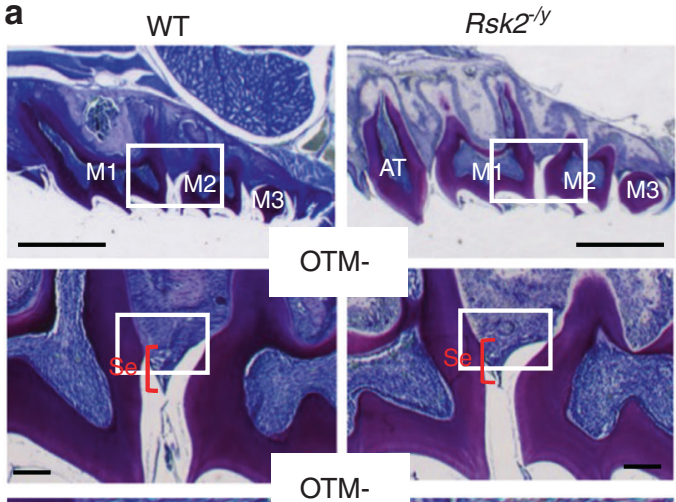

OTM-
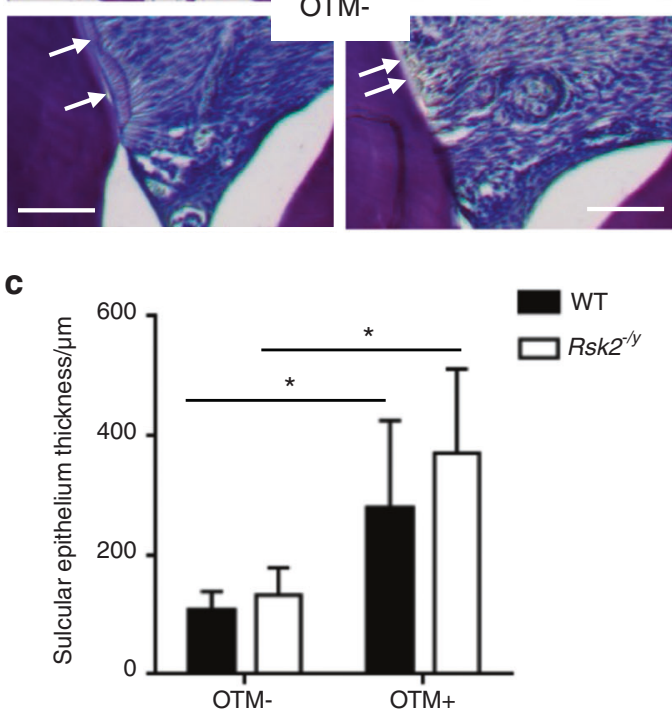

b

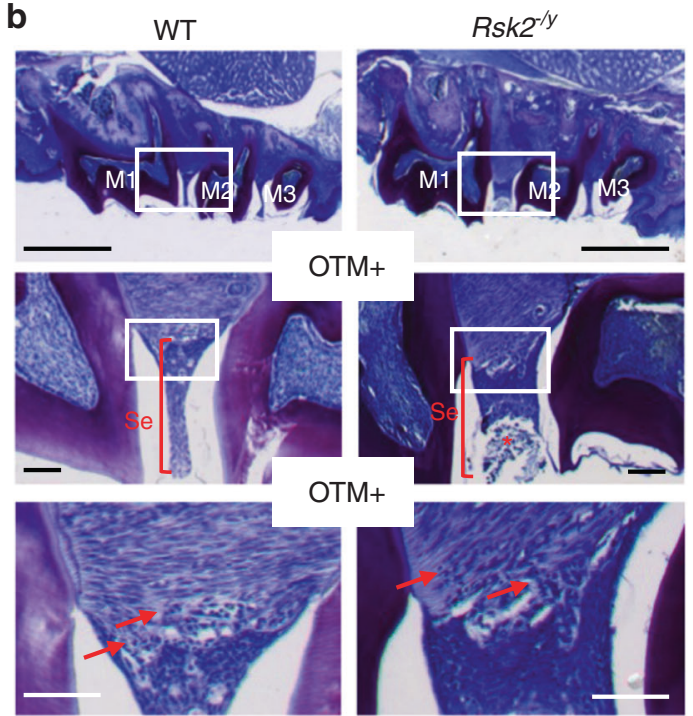

Fig. 2 Histological analysis of tooth movement in $R s k 2^{-1 y}$ mice and wild-type mice. $\mathbf{a}, \mathbf{b}$ Decalcified histological sections stained with toluidine blue of maxillary teeth from 12 weeks old $R s k 2^{-1 y}$ mice and WT mice. The areas indicated by the white boxes in the upper panels and middle panels are shown at higher magnifications in the middle and lower panels. The acellular cementum layer of teeth from $R s k 2^{-1 y}$ mice appears thinner as compared to those of WT mice (white arrows in a, lower panels). The OTM-induced approximal gap allows debris to accumulate (red asterisk in b, middle panels) causing inflammatory hyperplasia of the sulcular epithelium (se) with an influx of lymphocytes (red arrows in b, lower panels). Scale bars: upper panels are $1 \mathrm{~mm}$; middle and lower panels are $100 \mu \mathrm{m}$. c Quantification of thickness of the sulcular epithelium in $R s k 2^{-1 y}$ and WT mice. Values are means \pm SD. $(n=4-5) .{ }^{*} P<0.05$

after OTM (Fig. 3j). Taken together, our histological analysis revealed that OTM induces a characteristic periodontal response in $R s k 2^{-l y}$ mice that does not seem to differ from those of WT mice.

Cementum hypoplasia does not increase the amount of OTMinduced root resorption in $R s k 2^{-1 y}$ mice

We finally determined whether the loss of Rsk2 function may increase the risk of OTM-induced root resorption. We, therefore, first quantified cellular and acellular cementum of teeth that were not subjected to tooth movement in $R s k 2^{-/ y}$ and WT mice (Fig. 4a). Confirming our previous observations on mandibular teeth of $R s k 2^{-1 y}$ mice, ${ }^{18}$ we observed a significant reduction of cellular cementum area and acellular cementum thickness in $R s k 2^{-1 y}$ mice (Fig. 4b, c). Interestingly, we also found a significant reduction of the cellular cementum area after OTM in WT teeth (Fig. 4b). This OTM-induced reduction of cellular cementum was not observed in $R s k 2^{-/ y}$ mice. Although the overall thickness of acellular cementum was not affected by OTM in WT and Rsk2 $2^{-1 y}$ mice (Fig. 4c), the cementum layer was partially lost and resorption lacunae were evident (Fig. 4d). These resorptions extended in the dentin layer and were thus identified as lateral root resorption. The shape and the localization of these resorptions did not differ between WT and $R s k 2^{-1 y}$ mice. Our quantification of the percentage of root resorption per root surface on the compression side also revealed no significant differences between WT and $R s k 2^{-l y}$ mice (Fig. 4e). Taken together, these results indicate that cementum hypoplasia in $R s k 2^{-/ y}$ mice does not increase the amount of OTM-induced root resorption.

\section{DISCUSSION}

Our study confirms that RSK2 has a decisive influence on the structures of the periodontium and demonstrates, for the first time, that RSK2 plays an important role in OTM.

The typical dental finding diagnostic of CLS in children is the premature loss of teeth. ${ }^{14-17,28}$ This is partially phenocopied in Rsk2-deficient mice, which show an increased loss of alveolar bone. ${ }^{18}$ In this study, we also observed this horizontal alveolar bone loss around maxillary molars that were not subjected to tooth movement. However, it is unlikely that the reason for the alveolar bone loss in $R s k 2^{-1 y}$ mice is a systemic defect of osteoblasts as we used rather young (10-12 weeks old) mice for this study and the osteopenic phenotype of $R s k 2^{-1 y}$ mice develops at an older age. ${ }^{3}$ This was also documented by our $\mu$-CT analysis of calvarial and palatal bone where we did not observe any significant differences between $R s k 2^{-/ y}$ mice and WT littermates (Supplementary Fig. 1). Furthermore, quantitative backscattered 


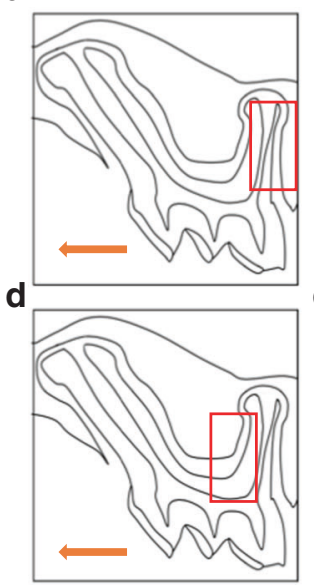

g

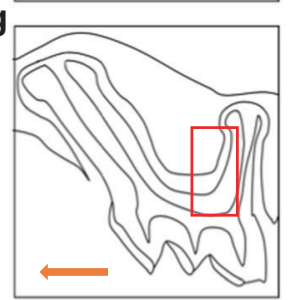

b

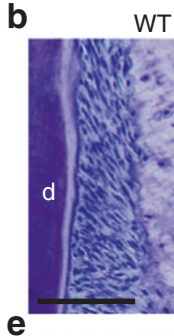

e

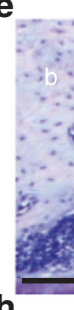

h

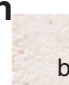

WT
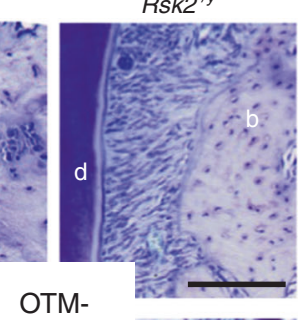

OTM-

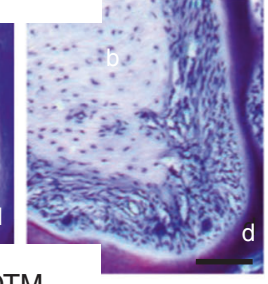

OTM-

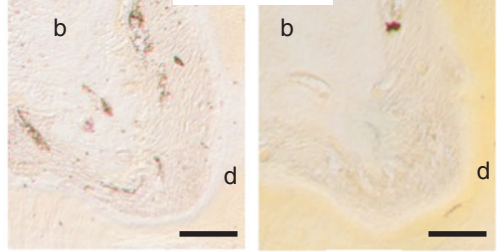

d

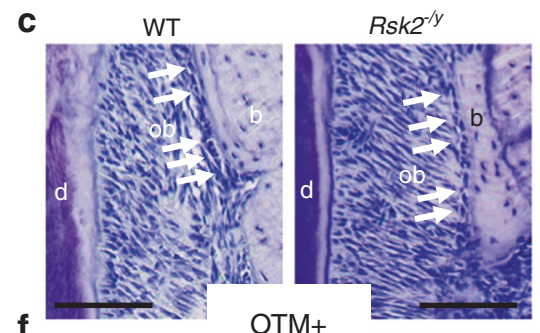

f

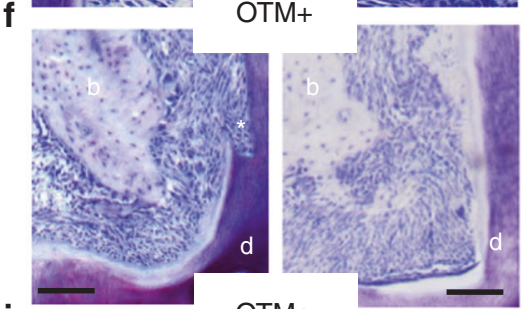

i

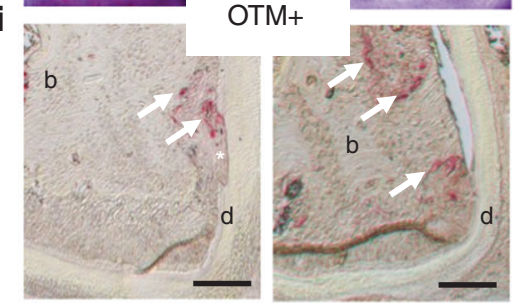

j

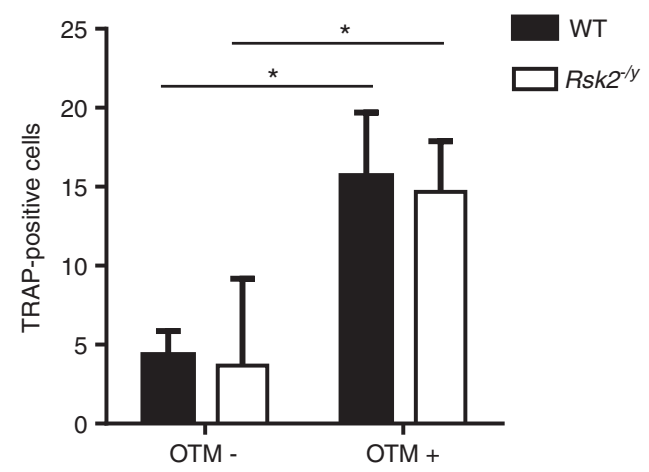

Fig. 3 Histological evaluation of OTM-induced pressure and tension zones. a-c Schematic drawing of a murine, maxillary M1. The tension side (red box in a) was analyzed on toluidin-blue stained sections of maxillary teeth from 12 weeks old Rsk2 $2^{-1 y}$ and WT mice. White arrows indicate osteoblasts. $\mathbf{d}$-f Toluidin-blue stained histological sections of the compression side from the same mice red box in $\mathbf{d}$. Root resorption were evident after OTM (white asterisks). g-i Immunohistochemical TRAP-stained histological sections of the compression side from the same mice red box in g. Numerous TRAP-positive cells are evident after OTM in Rsk2 $2^{-1 y}$ and WT mice (white arrows in i). j Quantification of TRAPpositive osteoclasts in WT and $R s k 2^{-1 /}$ mice. Values are means \pm SD. $(n=3)$. b, bone; d, dentin; ob, osteoblast. Scale bar $=100 \mu$ m. ${ }^{*} P<0.05$

electron imaging revealed normal mineralization of trabecular bone in $R s k 2^{-/ y}$ mice (Supplementary Fig. 2). This indicates that trabecular bone morphology and quantity is not significantly affected by loss of RSK2 function in young mice. It is, therefore, more likely that the alveolar bone loss is caused by a cellautonomous defect of the cementoblasts leading to dysfunctional acellular cementum formation as described previously. ${ }^{18}$

Accordingly, our histological analysis of maxillary molars revealed a reduced thickness of acellular cementum in $R s k 2^{-/ y}$ mice. The periodontal phenotype of $R s k 2^{-/ y}$ mice, therefore, seems to be comparable to that of BSP-deficient mice, in which hypoplasia of acellular cementum leads to periodontal dysfunction and subsequent to a reduced ability to resist mechanical forces, leading to alveolar bone loss. ${ }^{25,29-31}$

It is also certainly possible that RSK2, as a member of the MAPK pathway, affects signal transduction pathways during OTM. Indeed, several studies have demonstrated that the MAPK pathway plays an important role during OTM. ${ }^{32,33}$ For instance, it has been shown that stress applied to PDL cells activates downstream targets of the MAPK pathways such as ERK1/2 and p38. Interestingly, inhibition of these target genes can also decrease the expression of osteoblast markers such as ALP, OPN, and BSP. It is, therefore, possible that lack of RSK2 also causes a reduction of ALP, OPN, and BSP during OTM, as RSK2 acts as a downstream kinase of these pathways. Moreover, it is noteworthy that mechanical stimulation of human periodontal fibroblasts induced the expression of the RSK2 substrate ATF4, which acts as an important factor for osteoblast differentiation from PDL cells. ${ }^{34}$ Whereas our study cannot clarify the exact biological role of RSK2 during OTM, we clearly demonstrated that RSK2 does affect OTM in vivo.

Besides accelerated tooth movement we also observed that orthodontic forces in $R s k 2^{-/ y}$ mice significantly exacerbated the alveolar bone loss. ${ }^{31}$ Interestingly, dysfunctional cementum function in BSP-deficient mice also reduces the ability of the PDL to resist forces and leads to mechanical-induced further alveolar bone loss. ${ }^{31}$ However, this seems unlikely to be the case in $R s k 2^{-1 y}$ mice as the amount of OTM-induced bone loss was not 

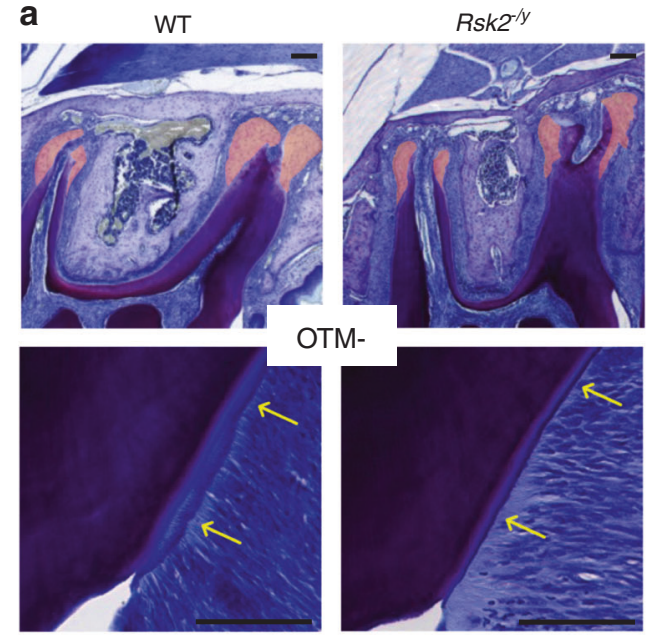

\section{M-}

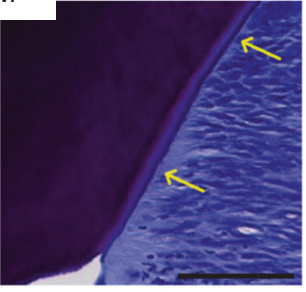

d

WT

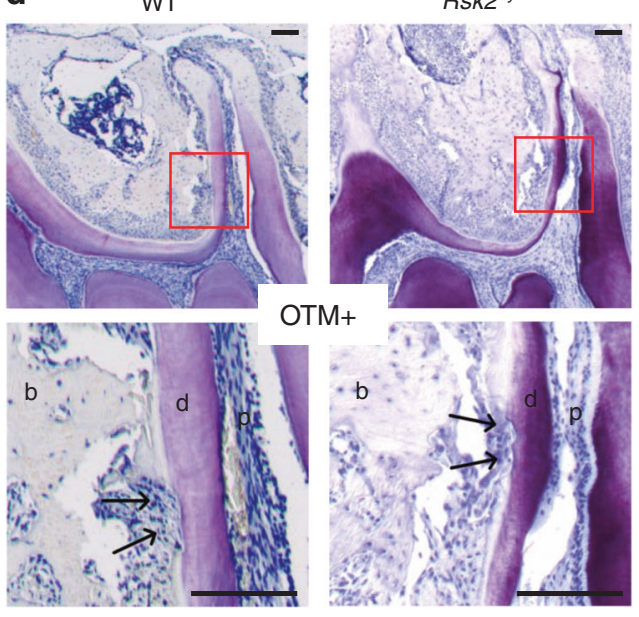

b
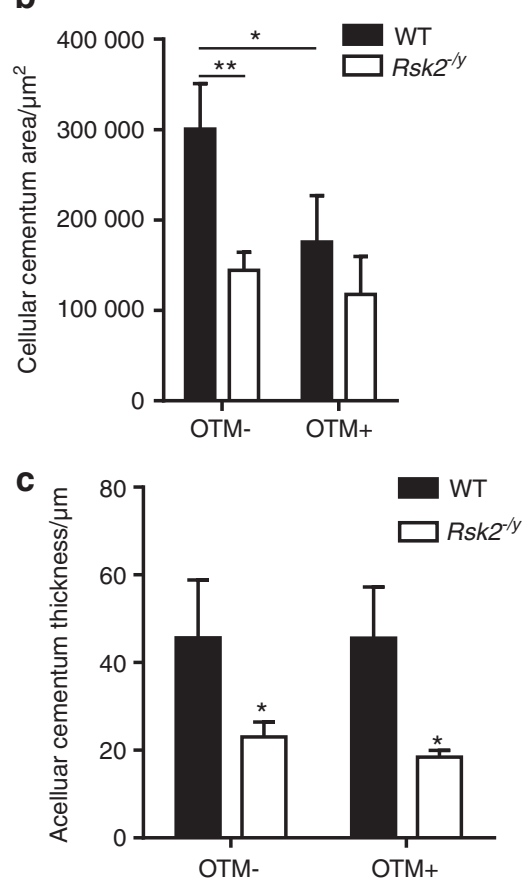

e

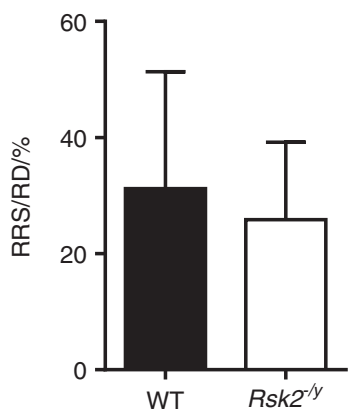

Fig. 4 Histological examination of the cementum layer and OTM-induced root resorptions. a Decalcified histological sections stained with toluidine blue of maxillary teeth from 12 weeks old $R s k 2^{-1 y}$ mice and WT mice. Orange area indicates cellular cementum and yellow arrows indicate acellular cementum. b Quantification of the area of cellular cementum and the thickness of acellular cementum in $\mathbf{c}$ in Rsk2 $2^{-1 y}$ and WT mice. Values are means $\pm \mathrm{SD}$. $\left(n=3-5,{ }^{*} P<0.05,{ }^{*} P<0.01\right)$. d Toluidine blue stained histological sections of the same mice showing root resorptions. The lower panels are magnifications of the areas depicted by the red rectangles in the upper panels. Black asterisks indicate root resorptions. b, bone; d, dentin; p, pulp. e Quantification of the resorbed root surface per root surface (RRS/RS) of 12 weeks old Rsk2 ${ }^{-1 y}$ ( $n=4$ ) and WT mice $(n=5)$. Scale bar $=100 \mu \mathrm{m}$. Values are means \pm SD

higher than those of control animals. We rather believe that debris niches due to the separation of the molars and the orthodontic appliance cause periodontitis and periodontal breakdown. In fact, our histological analysis of teeth after OTM revealed in both $R s k 2^{-l y}$ and WT mice clear signs of gingival inflammation apparent as significant swelling of sulcular epithelium and subepithelial lymphocyte influx. Another reason for the bone loss after OTM could be the application of supraphysiological forces. However, Taddei et al. described that the force of $0.35 \mathrm{~N}$, which we used in our study, is necessary to obtain tooth movement and causes relatively little tissue damage in mice. ${ }^{35}$ Our histological analysis indicated that the force level was not too high as we observed a normal cellular response to OTM in Rsk $2^{-1 y}$ and WT mice. This cellular response did not seem to differ between $R s k 2^{-/ y}$ and WT mice. In fact, both groups showed a significant increase in TRAPpositive osteoclasts after OTM, a distinct sign of bone remodeling.

Another important histological finding was that only a few areas of pressure-induced tissue necrosis (i.e., hyalinization) were evident in $R s k 2^{-1 y}$ and WT (data not shown). Small areas of hyalinization are almost unavoidable during OTM ${ }^{31,36}$ and only large areas of hyalinization would have been indicative for excessive forces. Also, the presence of root resorption in $R s k 2^{-/ y}$ and WT mice should be considered as a normal histological sign of OTM. $^{37}$

The reasons for the occurrence of orthodontic root resorption are far from understood but recent studies suggest that genetics plays a decisive role in its development. ${ }^{25-27,29,30}$ In this regard, it was of particular interest for us to analyze the role of RSK2 in the development of root resorption. Since acellular cementum has a protective function against lateral root resorptions, ${ }^{25-27,38}$ we assumed that a reduced layer of acellular cementum in $R s k 2^{-1 y}$ mice leads to more root resorptions; ${ }^{18}$ however, this could not be confirmed in our study. No significant differences were observed between $R s k 2^{-1 y}$ mice and WT mice with regard to the percentage of resorptions in relation to the root surface, or to the shape and localization of the resorptions. It, therefore, seems that also a reduced layer of acellular cementum may protect against root resorption as long as the integrity of the cementum layer is preserved. Another reason for the small amount of root resorption in $R s k 2^{-1 y}$ mice could be the alveolar bone phenotype. ${ }^{26,39-41}$ In fact, as root resorptions occur more frequently in areas of high tissue pressure ${ }^{40,41}$ it is possible that the alveolar bone loss of 
$R s k 2^{-/ y}$ mice causes less resistance to OTM. This does not only seem to accelerate OTM but may also decrease the risk of root resorptions. Nevertheless, we can conclude that loss of RSK2 function is not a risk factor for the development of OTM-induced root resorptions. This does not imply that loss of RSK2 function may not affect the repair of root resorptions, which seems to be governed by the same molecular mechanism that also controls cementogenesis during tooth development. ${ }^{20,21,39,41,42}$ Since loss of RSK2 function negatively affects cementogenesis, the repair of root resorptions may be impaired in $R s k 2^{-/ y}$ mice. This should be addressed in futures studies.

The unique dental phenotype of $R s k 2^{-/ y}$ mice caused some limitations in the experimental setup. The presence of small, supernumerary teeth mesial to the $\mathrm{M} 1$ in $R s k 2^{-1 y}$ mice was striking and confirmed previous observations. ${ }^{43,44}$ It seems that the loss of RSK2 function leads to the reappearance of diastemal teeth, a type of tooth that was lost in rodents during evolution. ${ }^{43}$ As these diastemal teeth may affect tooth movement it was important to exclude mice showing bilateral a supernumerary tooth in the upper dentition from our experiments. The number of animals per group in our study is therefore comparatively low and it is possible that more significant differences would have been found had more mice been used.

Despite these difficulties, our results demonstrate that our orthodontic mouse model is appropriate for analyzing the role of individual genes during OTM and the development of root resorptions in vivo.

\section{CONCLUSION}

Taken together, we can conclude that RSK2 plays a nonredundant role in OTM. Loss of Rsk2 function accelerates tooth movement without causing more root resorptions, although $R s k 2^{-/ y}$ mice exhibit reduced levels of acellular cementum. We, therefore, believe that the relationship between root cementum and orthodontic root resorption is less clear than previously expected. Future studies should use transgenic mice to decipher the genetic interplay between cementogenesis and orthodontic root resorptions.

\section{MATERIALS AND METHODS}

Mice

Rsk2-deficient mice $\left(R s k 2^{-/ y}\right)^{3,4}$ and control wild-type littermates (WT) were maintained at the animal facility of the University Medical Center Hamburg-Eppendorf on a C57BL/6J background. The generation of $R s k 2^{-\not y}$ mice by homologous recombination has been described previously. ${ }^{4}$ Briefly, the targeting vector was located in exon 2 of the Rsk2 gene (Rps6ka3) and contained a neomycin resistance gene, followed by three stop codons and flanked by two loxP sites. The construct was electroporated in ES cells and, after NeoR selection, injected into C57BL/6J blastocysts. The NeoR cassette was finally removed by crossing with a C57BL/ $6 J / C M V-C r e ~ t r a n s g e n i c ~ l i n e . ~ R s k 2^{-/ y}$ mice are viable and show reduced body weight (Supplementary Fig. 3). The bone phenotype of $R s k 2^{-/ y}$ mice was the subject of several studies and is characterized by osteopenia due to osteoblast dysfunction. 3,4,45 $R s k 2^{-/ y}$ mice additionally exhibit craniofacial dysmorphia and alveolar bone loss due to cementum defects. ${ }^{18,43}$ Due to the $x$ chromosomal inheritance of the Rsk2 gene only male animals were used in this study. All experiments were carried out on 4-5 mice per genotype at 10 weeks of age at the time of the orthodontic procedure. The weight of each animal was measured over the entire period of the experiments. In both groups there was a slight, but not significant, reduction of body weight in the first three days after anesthesia (Supplementary Fig. 3). The animals were subjected to a normal $12 \mathrm{~h}$ light/dark rhythm at a constant temperature of $24^{\circ} \mathrm{C}$. Furthermore, they were kept on a soft food diet consisting of mashed dry food pellets to prevent mechanical damage to the orthodontic appliance. All animal procedures were performed under consent with the commission for animal welfare (Behörde für Gesundheit und Verbraucherschutz der Hansestadt Hamburg, Nr. 072/18).

\section{Orthodontic appliance}

The experiment was carried out as a split-mouth model in which the untreated side of the jaw served as an internal control. ${ }^{46}$ The mice were anesthetized with a solution of ketamine $\left(40 \mathrm{mg} \cdot \mathrm{kg}^{-1}\right.$ bw Ketamin-S), xylazine $2 \%\left(16 \mathrm{mg} \cdot \mathrm{kg}^{-1} \mathrm{bw}\right)$, and heparin (40 000 IE per kg bw in $0.9 \% \mathrm{NaCl})\left(10 \mathrm{~mL} \cdot \mathrm{kg}^{-1}\right)$. OTM was not performed in Rsk2-deficient mice that showed an additional tooth mesial to the $M 1 .{ }^{17,43}$ The anesthetized mice were carefully fixed on a treatment lathe (Unimat 3, Emco, Wiener Neudorf, Austria). The maxillary incisors were then attached to the operating table with a loop of suture material (Vicryl, Ethion Inc., New Jersey, USA) (Fig. $1 \mathrm{a})$, and the incisors of the mandible were fixed with an orthodontic rubber chain (Elasto-force, Dentaurum, Ispringen, Germany). All procedures were performed using magnifying glasses (Zeiss G 2.5 TL, Carl Zeiss Germany, Oberkochen, Germany) and a headlamp (Sigma Smart study, Sigma Dental Systems, Handewitt, Germany). The surface of the left first maxillary molar and incisors were etched with $37 \%$ phosphoric acid gel (HS-etch gel 37\%, Henry Schein Dental, Langen, Germany). The bonding (Scotchbond, $3 \mathrm{~m}$ Espe, Neuss, Germany) was applied after removal of the etching gel and sufficient drying of the surface. The distal end of the Nitinol open coil spring (Sentalloy Open Coil Spring, Dentsply Sirona, Pennsylvania, USA) was bonded to the surface of the left first maxillary molar with a light-cured resin (Estelite Flow Quick, Tokuyama Dental Corp., Tokyo, Japan). The treatment table was moved backward, parallel to the direction of the force, to achieve the desired activation of 35 centinewton that was controlled with a tension gauge (tension gauge, Dentaurum, Ispringen, Germany). With achieving the desired force the anterior part of the coil spring was then bonded to both incisors (Fig. 1a, b). The animals were monitored on a warming mat after the orthodontic procedure until they woke up from anesthesia. After 12 days of OTM, the animals were euthanized by $\mathrm{CO}_{2}$ inhalation. The heads were fixed for $24 \mathrm{~h}$ in formalin (Formafix 3,5\%, Grimm med Logistik GmbH, Torgelow, Germany) and then transferred to $80 \%$ ethanol.

Contact radiography and micro-computed tomography imaging The skulls were radiographed in a digital contact radiography cabinet (Faxitron X-ray Corp., Wheeling, IL, USA) (Fig. 1c) and scanned with a voxel size of $15 \mu \mathrm{m}$. Micro-CT images $(\mu \mathrm{CT} 40$ Scanco Medical, Bassersdorf, Switzerland) were acquired at $55 \mathrm{kVp}$ with an anode current of $145 \mu \mathrm{A}$ and an integration time of $200 \mathrm{~ms}$. The reconstruction of the raw data images was performed with the scanco $\mu C T$ evaluation program V6.6. Analysis of $\mu C T$ images was performed using ImageJ 1.52 (National Institutes of Health, Bethesda, MD, USA) and its implication BoneJ 1.4.3. ${ }^{47}$ The area of bone loss was pseudo-colored with Photoshop Cs4 (Adobe Systems Inc., Mountain View, CA, USA).

Histology and histomorphometry

After performed $\mu \mathrm{CT}$-analysis the upper jaws were prepared for histological examination. They were decalcified using Usedcalc (MEDITE Medical GmbH, Burgdorf, Germany), an EDTA containing reagent, for 21 days, while the reagent was changed once a week. Afterward, the upper jaws were cut in the parasagittal plane. The halves were dehydrated in ascending alcohol concentrations and embedded in paraffin. Sections of $4 \mu \mathrm{m}$ thickness were cut using a microtome (Supercut 2050, Reichert-Jung, Leica Microsystems $\mathrm{GmbH}$, Wetzlar, Germany) and stained with toluidine blue. For this, the sections were deparaffined in xylene baths $(3 \times 5 \mathrm{~min})$. The samples were rehydrated in descending alcohol series for 2 min each, followed by a short wash in distilled water. Afterward, they 
a
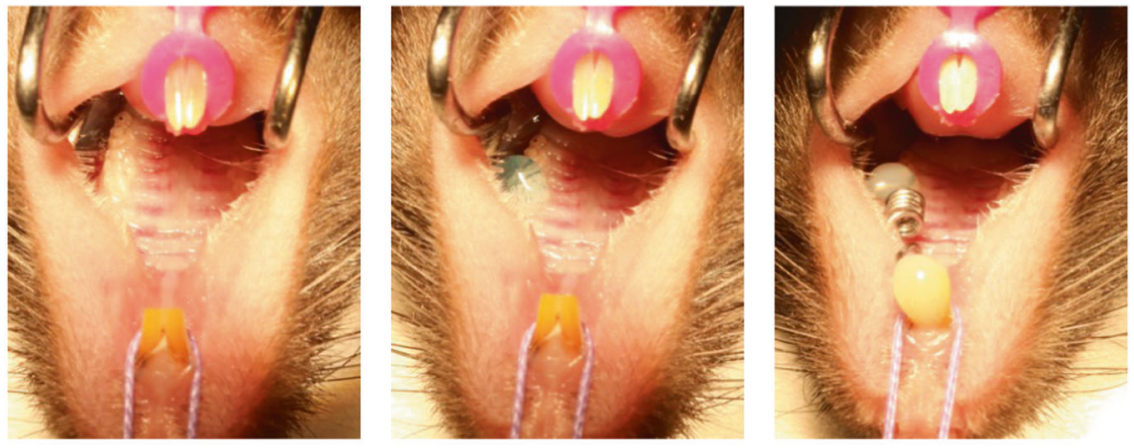

b

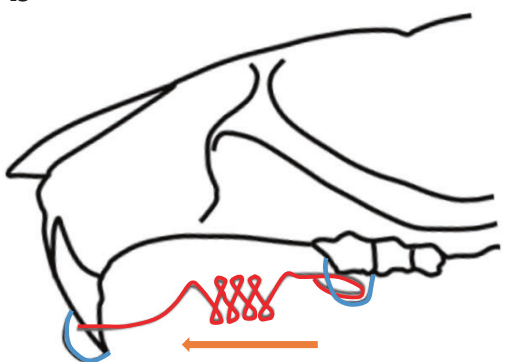

C

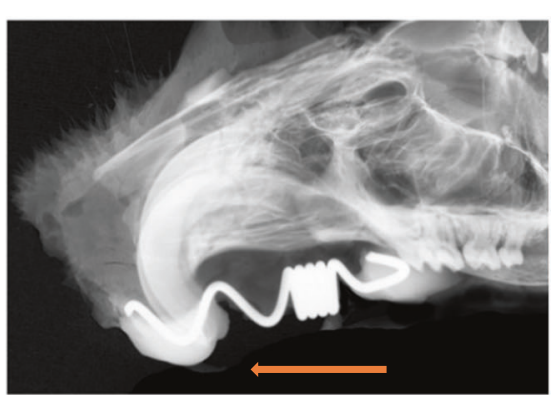

Fig. 5 Description of the experimental setup. a Photographs of the procedure. The upper and lower incisors are attached to the table using a suture and rubber chain (left picture). The teeth were etched with $37 \%$ phosphoric acid gel (middle picture) and the coil spring was then fixed with light-curing resin to the first molar and the incisors (right picture). b Illustration of the OTM model and contact radiography of the appliance in c. The NiTi-tension spring (red) was attached to the incisor and the first molar using a dental light-curing resin (blue). The orange arrows indicate the mesial direction of the force

were dyed in $1 \%$ toluidine blue $(\mathrm{pH} 4.5)$ for $30 \mathrm{~min}$. The stained sections were then rinsed with distilled water and in the next step with $80 \%$ ethanol, followed again by a short wash in distilled water, in order to dehydrate them in an ascending alcohol series. Before covering with Eukitt (ORSAtec $\mathrm{GmbH}$, Bobingen, Germany), an infiltration with xylene was executed in three consecutive baths for 5 min each. TRAP staining was performed as follows: the sections were dewaxed in xylene $(3 \times 5 \mathrm{~min})$ and rehydrated in a descending alcohol series as described for the toluidine blue staining. Totally, $50 \mathrm{~mL}$ buffer solution is prepared from $40 \mathrm{mmol} \cdot \mathrm{L}^{-1}$ sodium acetate (pH 5) and $10 \mathrm{mmol} \cdot \mathrm{L}^{-1}$ sodium tartrate. This is mixed with $5 \mathrm{mg}$ Naphthol-AS-MX phosphate dissolved in $500 \mu \mathrm{L}$ dimethylformamide. Then $30 \mathrm{mg}$ Fast Red Violet is added and homogenized while shaking. The prepared sections get immersed in the solution for about $120 \mathrm{~min}$ and incubated at $37^{\circ} \mathrm{C}$. The samples were not counterstained and covered with Aquatex (Merck KGaA, Darmstadt, Germany) in the end.

All histomorphometric analyses were done using the OsteoMeasure histomorphometry system (Osteometrics, Atlanta, GA, USA). Cellular cementum was quantified at both root apices of the first maxillary molar and the thickness of acellular cementum was quantified along its full length on the distal root surface of the first maxillary molar as previously described. ${ }^{18}$

Quantitative backscattered electron imaging (qBEl)

Methylmethacrylate-embedded specimens of vertebrae from 12week-old WT and $R s k 2^{-1 y}$ mice were polished and carbon-coated. Afterward, they were mounted in a scanning electron microscope (LEO 435 VP; LEO Electron Microscopy Ltd., Cambridge, England) with a backscattered electron detector (Type 202, K.E. Developments Ltd., Cambridge, England). To determine the mineralization of the specimens they were scanned at $20 \mathrm{kV}$ and a 680-pA electron beam current. Images were acquired at $20 \times$ and $150 \times$ magnification and analyzed using a customized MATLAB (TheMathWorks, Inc.
Natick, Massachusetts, USA) script. Generated gray values were transferred in the mean calcium content. For each specimen the mean calcium content ( $\mathrm{Ca}$ Mean) and the mineralization heterogeneity (Ca Width) were analyzed. In each individual, three images per vertebrae were considered for the qBEl evaluations (Fig. 5).

\section{Statistical analysis}

The statistical analysis was performed with GraphPad PRISM6 (GraphPad Software, San Diego, USA). We analyzed the data for normal distribution using the Shapiro-Wilk test. Results were expressed as the mean \pm SD. Two-sided $t$ test and one-way ANOVA with Bonferroni post hoc test were used for multiple group comparisons. $P$ values below 0.05 were considered statistically significant.

\section{ACKNOWLEDGEMENTS}

This work is in memory of Dr. Jean-Pierre David, a brilliant bone biologist, who inspired this and many other projects. Open Access funding enabled and organized by Projekt DEAL.

\section{ADDITIONAL INFORMATION}

The online version of this article (https://doi.org/10.1038/s41368-020-00102-4) contains supplementary material, which is available to authorized users.

Competing interests: The authors declare no competing interests.

\section{REFERENCES}

1. Nebreda, A. R. \& Gavin, A. C. Cell survival demands some Rsk. Science 286, 1309-1310 (1999).

2. Romeo, Y., Zhang, X. \& Roux, P. P. Regulation and function of the RSK family of protein kinases. Biochem. J. 441, 553-569 (2012).

3. David, J. P. et al. Essential role of RSK2 in c-Fos-dependent osteosarcoma development. J. Clin. Investig. https://doi.org/10.1172/JCI200522877 (2005). 
4. Yang, X. et al. ATF4 is a substrate of RSK2 and an essential regulator of osteoblast biology: implication for Coffin-Lowry syndrome. Cell 117, 387-398 (2004).

5. Xing, J., Ginty, D. D. \& Greenberg, M. E. Coupling of the RAS-MAPK pathway to gene activation by RSK2, a growth factor-regulated CREB kinase. Science 273, 959-963 (1996).

6. Brüning, J. C. et al. Ribosomal subunit kinase-2 is required for growth factorstimulated transcription of the c-Fos gene. Proc. Natl Acad. Sci. USA 97, 2462-2467 (2000).

7. Zhang, R. et al. Transcriptional regulation of BMP2 expression by the PTH-CREB signaling pathway in osteoblasts. PLoS ONE 6, e20780 (2011).

8. Huang, W. C. et al. Human osteocalcin and bone sialoprotein mediating osteomimicry of prostate cancer cells: role of CAMP-dependent protein kinase A signaling pathway. Cancer Res. 65, 2303-2313 (2005).

9. $\mathrm{Yu}, \mathrm{S}$. et al. ATF4 promotes á-catenin expression and osteoblastic differentiation of bone marrow mesenchymal stem cells. Int. J. Biol. Sci. 9, 256-266 (2013).

10. $\mathrm{Yu}, \mathrm{S}$. et al. Critical role of activating transcription factor 4 in the anabolic actions of parathyroid hormone in bone. PLoS ONE 4, e7583 (2009).

11. Almeida, M. et al. Estrogen receptor-a signaling in osteoblast progenitors stimulates cortical bone accrual. J. Clin. Investig. 123, 394-404 (2013).

12. Xiao, G. et al. Cooperative interactions between activating transcription factor 4 and Runx2/Cbfa1 stimulate osteoblast-specific osteocalcin gene expression. J. Biol. Chem. 280, 30689-30696 (2005).

13. Wang, Z. Q. et al. Bone and haematopoietic defects in mice lacking c-fos. Nature 360, 741-745 (1992)

14. Norderyd, J. \& Aronsson, J. Hypoplastic root cementum and premature loss of primary teeth in Coffin-Lowry syndrome: a case report. Int. J. Paediatr. Dent. 22, 154-156 (2012).

15. Igari, K., Hozumi, Y., Monma, Y. \& Mayanagi, H. A case of Coffin-Lowry syndrome with premature exfoliation of primary teeth. Int. J. Paediatr. Dent. 16, 213-217 (2006).

16. Day, P., Cole, B. \& Welbury, R. Coffin-Lowry syndrome and premature tooth loss: a case report. J. Dent. Child. 67, 148-150 (2000).

17. Hanauer, A. \& Young, I. D. Coffin-Lowry syndrome: clinical and molecular features. J. Med. Genet 39, 705-713 (2002).

18. Koehne, T. et al. Rsk2, the kinase mutated in Coffin-Lowry syndrome, controls cementum formation. J. Dent. Res. 95, 752-760 (2016).

19. Hartsfield, J. K., Everett, E. T. \& Al-Qawasmi, R. A. Genetic factors in external apical root resorption and orthodontic treatment. Crit. Rev. Oral. Biol. Med. 15, 115-122 (2004).

20. Al-Qawasmi, R. A. et al. Root resorption associated with orthodontic force in inbred mice: genetic contributions. Eur. J. Orthod. 28, 13-19 (2006).

21. Al-Qawasmi, R. A. et al. Genetic predisposition to external apical root resorption in orthodontic patients: linkage of chromosome-18 marker. J. Dent. Res. 82, 356-360 (2003).

22. Weltman, B., Vig, K. W. L., Fields, H. W., Shanker, S. \& Kaizar, E. E. Root resorption associated with orthodontic tooth movement: a systematic review. Am. J. Orthod. Dentofac. Orthop. 137, 462-476 (2010).

23. Harris, E. F., Kineret, S. E. \& Tolley, E. A. A heritable component for external apical root resorption in patients treated orthodontically. Am. J. Orthod. Dentofac. Orthop. 111, 301-309 (1997).

24. Ngan, D. C. S., Kharbanda, O. P., Byloff, F. K. \& Darendeliler, M. A. The genetic contribution to orthodontic root resorption: a retrospective twin study. Aust. Orthod. J. 20, 1-9 (2004)

25. B. L., F. et al. Deficiency in acellular cementum and periodontal attachment in Bsp null mice. J. Dent. Res. 92, 166-172 (2013).

26. Bosshardt, D. D. Are cementoblasts a subpopulation of osteoblasts or a unique phenotype?. J. Dent. Res. 84, 390-406 (2005).

27. Iglesias-Linares, A. \& Hartsfield, J. K. Cellular and molecular pathways leading to external root resorption. J. Dent. Res. 96, 145-152 (2017).

28. Temtamy, S. A., Miller, J. D. \& Hussels-Maumenee, I. The Coffin-Lowry syndrome: an inherited faciodigital mental retardation syndrome. J. Pediatr. 86, 724-731 (1975).

29. Foster, B. L. et al. Mineralization defects in cementum and craniofacial bone from loss of bone sialoprotein. Bone 78, 150-164 (2015).
30. Foster, B. et al. Tooth root dentin mineralization defects in a mouse model of hypophosphatasia. J. Bone Miner. Res. 28, 271-282 (2013).

31. Meikle, M. C. The tissue, cellular, and molecular regulation of orthodontic tooth movement: 100 years after Carl Sandstedt. Eur. J. Orthod. 28, 221-240 (2006).

32. Papadopoulou, A., Iliadi, A., Eliades, T. \& Kletsas, D. Early responses of human periodontal ligament fibroblasts to cyclic and static mechanical stretching. Eur. J. Orthod. 39, 258-263 (2017).

33. Jiang, L. \& Tang, Z. Expression and regulation of the ERK $1 / 2$ and p38 MAPK signaling pathways in periodontal tissue remodeling of orthodontic tooth movement. Mol. Med. Rep. 17, 1499-1506 (2018).

34. Wei, F. et al. The effect of centrifugal force on the mRNA and protein levels of ATF4 in cultured human periodontal ligament fibroblasts. Arch. Oral Biol. 53, 35-43 (2008).

35. Rodrigues De Albuquerque Taddei, S. et al. Experimental model of tooth movement in mice: a standardized protocol for studying bone remodeling under compression and tensile strains. J. Biomech. 45, 2729-2735 (2012).

36. Löe, H. \& Waerhaug, J. Experimental replantation of teeth in dogs and monkeys. Arch. Oral. Biol. 3, 176-184 (1961).

37. Killiany, D. M. Root resorption caused by orthodontic treatment: an evidencebased review of literature. Semin. Orthod. 5, 128-133 (1999).

38. Chung, C. J. et al. OPN deficiency suppresses appearance of odontoclastic cells and resorption of the tooth root induced by experimental force application. J. Cell. Physiol. 214, 614-620 (2008).

39. Matsumoto, T., limura, T., Ogura, K., Moriyama, K. \& Yamaguchi, A. The role of osteocytes in bone resorption during orthodontic tooth movement. J. Dent. Res. 92, 340-350 (2013).

40. Cuoghi, O. A., Aiello, C. A., Consolaro, A., Tondelli, P. M. \& Mendonça, M. R. de. Resorption of roots of different dimension induced by different types of forces. Braz. Oral. Res. 28, 1-7 (2014).

41. Langford, S. R. \& Sims, M. R. Root surface resorption, repair, and periodontal attachment following rapid maxillary expansion in man. Am. J. Orthodont. 81, 108-115 (1982)

42. Yoshimatsu, M. et al. Experimental model of tooth movement by orthodontic force in mice and its application to tumor necrosis factor receptor-deficient mice. J. Bone Mineral Metab. 24, 20-27 (2006).

43. Laugel-Haushalter, V. et al. RSK2 is a modulator of craniofacial development. PLoS ONE 9, e84343 (2014).

44. Marangoni, P. et al. Phenotypic and evolutionary implications of modulating the ERK-MAPK cascade using the dentition as a model. Sci. Rep. 5, 11658 (2015).

45. Böhm, C. et al. RSK2 protects mice against TNF-induced bone loss. J. Cell Sci. 125, 2160-2171 (2012).

46. Decker, M. G. et al. Role of c-Fos in orthodontic tooth movement: an in vivo study using transgenic mice. Clin. Oral Investig. https://doi.org/10.1007/s00784-02003503-1 (2020).

47. Doube, M. et al. BoneJ: free and extensible. Bone 47, 1076-1079 (2010).

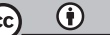

Open Access This article is licensed under a Creative Commons Attribution 4.0 International License, which permits use, sharing, adaptation, distribution and reproduction in any medium or format, as long as you give appropriate credit to the original author(s) and the source, provide a link to the Creative Commons license, and indicate if changes were made. The images or other third party material in this article are included in the article's Creative Commons license, unless indicated otherwise in a credit line to the material. If material is not included in the article's Creative Commons license and your intended use is not permitted by statutory regulation or exceeds the permitted use, you will need to obtain permission directly from the copyright holder. To view a copy of this license, visit http://creativecommons. org/licenses/by/4.0/.

(c) The Author(s) 2020 